\title{
Accuracy of aortic root annulus assessment with cardiac magnetic resonance in patients referred for transcatheter aortic valve implantation: a comparison with multi-detector computed tomography
}

\author{
Gianluca Pontone*, Daniele Andreini, Erika Bertella, Saima Mushtaq, Sarah Cortinovis, Andrea Annoni, \\ Alberto Formenti, Giovanni Ballerini, Mauro Pepi
}

From 16th Annual SCMR Scientific Sessions

San Francisco, CA, USA. 31 January - 3 February 2013

\section{Background}

To compare the accuracy of cardiac magnetic resonance (CMR) evaluation of the aortic root as compared to multidetector computed tomography (MDCT) in patients referred for transcatheter aortic valve implantation (TAVI).

\section{Methods}

In 50 patients, the following parameters were assessed with CMR and compared with those obtained with MDCT: aortic annulus (AoA) maximum diameter (AoA-Dmax), minimum diameter (AoA-Dmin), and area (AoA-A), length of the left coronary, right coronary, and non-coronary aortic leaflets, degree (grades 1 to 4) of aortic leaflet calcification and distance between AoA and coronary artery ostia.

\section{Results}

AoA-Dmax, AoA-Dmin and AoA-A were $26.45 \pm 2.83 \mathrm{~mm}$, $20.17 \pm 2.20 \mathrm{~mm}, 444.88 \pm 84.61 \mathrm{~mm} 2$ and $26.45 \pm 2.76 \mathrm{~mm}$, $20.59 \pm 2.35 \mathrm{~mm}$ and $449.78 \pm 86.22 \mathrm{~mm} 2$ by MDCT and $\mathrm{CMR}$, respectively. The length of left coronary, right coronary, and non-coronary leaflets were $14.02 \pm 2.27 \mathrm{~mm}$, $13.33 \pm 2.33 \mathrm{~mm}, 13.39 \pm 1.97 \mathrm{~mm}$, and $13.95 \pm 2.18 \mathrm{~mm}$, $13.30 \pm 2.14 \mathrm{~mm}, 13.46 \pm 1.80 \mathrm{~mm}$ by MDCT and CMR, respectively, while the scores of aortic leaflet calcifications were $3.4 \pm 0.7$ vs. $2.97 \pm 0.77$. Finally, the distance between AoA and left main and right coronary artery ostia was
$16.21 \pm 3.07 \mathrm{~mm}, 16.02 \pm 4.29 \mathrm{~mm}$ and $16.14 \pm 2.83 \mathrm{~mm}$, $16.14 \pm 4.36 \mathrm{~mm}$ by CCT and CMR, respectively. There was close agreement between CMR and MDCT measurements, whereas aortic leaflet calcifications were underestimated by CMR.

\section{Conclusions}

Aortic root assessment with CMR including AoA size, aortic leaflet length and coronary artery ostia height is accurate in comparison to MDCT. CMR may be a valid imaging alternative in patients unsuitable for MDCT.

\section{Funding}

None.

Published: 30 January 2013

\section{doi:10.1186/1532-429X-15-S1-012}

Cite this article as: Pontone et al:: Accuracy of aortic root annulus assessment with cardiac magnetic resonance in patients referred for transcatheter aortic valve implantation: a comparison with multidetector computed tomography. Journal of Cardiovascular Magnetic Resonance 2013 15(Suppl 1):012.

Centro Cardiologico Monzino IRCCS, Milan, Italy 